\title{
Is emergency percutaneous antegrade drainage of the upper urinary tract useful for future percutaneous nephrolithotomy access?
}

\author{
Itay M. Sabler ${ }^{1}$, loannis Katafigiotis ${ }^{1,2}$, Stavros Sfoungaristos ${ }^{1,2}$, Amitay Lorber ${ }^{1}$, Ioannis Leotsakos ${ }^{1}$, \\ Vladimir Yutkin ${ }^{1}$, Guy Hidas ${ }^{1}$, Ofer N. Gofrit ${ }^{1}$, Mordechai Duvdevani ${ }^{1}$ \\ ${ }^{1}$ Department of Urology, Hadassah Hebrew University Medical Center, Jerusalem, Israel, ${ }^{2}$ Athens Stone Clinic, Athens, Greece
}

Purpose: To compare percutaneous nephrolithotomy (PCNL) operations between patients with a preoperative nephrostomy tube and patients that the renal access was obtained at the time of the surgery.

Materials and Methods: We retrospectively evaluated PCNL cases. Patients were divided into two groups. Group 1 - a non-nephrostomy tube (percutaneous nephrostomy, PCN) group and Group 2 - patients with a PCN placed before the procedure. All preoperatively placed PCN's were performed in emergency situations by interventional radiologists (IR). Complications were classified according to the Clavien-Dindo classification. We compared stone characteristics, operation time, complications, efficacy and PCN usability at surgery.

Results: Five hundred twenty-seven patients who were submitted to PCNL for renal stones were included in the study. In 73 patients (13.9\%) the PCNs were placed before the surgery. Patients and stone characteristics, mean operative time $(p=0.830)$, complications $(\mathrm{p}=0.859)$ and stone-free rates $(93.0 \%)$ were similar between the groups. There was a trend toward higher complication rates in Group 1, but the difference was not statistically significant. Only 21 (29.0\%) of preoperatively placed PCNs were used during PCNL for establishing a tract. The reasons for not using PCN tract were: pelvic or infundibular insertion (30.0\%) and suboptimal anatomic location (70.0\%).

Conclusions: Preoperative emergency inserted PCNs by IR usage rates were low during PCNL. Its placement neither affects the incidence of complications nor affects the operation time and outcomes. As such, when emergency renal drainage is indicated, the need for a future definitive PCNL should not influence the decision about the modality of renal drainage.

Keywords: Nephrostomy; Percutaneous nephrolithotomy; Renal lithiasis; Urolithiasis

This is an Open Access article distributed under the terms of the Creative Commons Attribution Non-Commercial License (http://creativecommons.org/licenses/by-nc/4.0) which permits unrestricted non-commercial use, distribution, and reproduction in any medium, provided the original work is properly cited.

\section{INTRODUCTION}

Percutaneous nephrolithotomy (PCNL) is an established treatment for staghorn calculi, large renal stone burden, lower pole stones, cysteine stones and stones associated with urinary tract abnormalities [1]. The most important step of PCNL, is tract planning and preparation, by choosing the suitable calyx, that enables the best exposure to the stone

Received: 30 September, 2018 - Accepted: 13 December, 2018

Corresponding Author: Ioannis Katafigiotis

Department of Urology, Hadassah Hebrew University Medical Center, Jerusalem 91120, Israel

TEL: +972-50-894-6816, FAX: +00302104522435, E-mail: katafigiotis.giannis@gmail.com

ORCID: https://orcid.org/0000-0002-2387-8604 
burden while minimizing surgical complications [2,3].

Percutaneous nephrostomy (PCN) still plays a key role in the decompression of the collecting system in acute septic clinical scenarios caused by upper urinary tract obstruction [4]. In these cases, they are mostly placed by interventional radiologists (IR). On the contrary, in the majority of PCNL cases, the primary renal access is obtained by a urologist during the operation, since the surgeon tries to perform the safest and more efficient access according to preoperative planning [5]. There are not a lot of data concerning the usability of a pre placed nephrostomy tube prior a PCN, but the most recent literature studies refer that the nephrostomy tube did not result in a decreased incidence of complications and at the same time did not contribute to increased success rates [6].

We retrospectively examined the usability, efficacy, and safety of emergency placed PCN as a possible tract for the PCNL access sheath placement.

\section{MATERIALS AND METHODS}

We retrospectively evaluated the data of patients submitted to PCNL from July 2011 to July 2016 in a high volume academic stone center. The IRB of the study is 0300-15-HMO (Department of Urology, Hadassah Hebrew University Medical Center, Jerusalem, Israel). Five hundred twenty-seven patients underwent PCNL for renal stones. Patients were stratified to two groups (Group 1 and Group 2) according to the obtained percutaneous access to the collecting system. In Group 1, the percutaneous access was made by a urologist during the operation to patients naïve to any preoperatively percutaneous access (emergency or not). Group 2 consisted of patients that underwent PCNL with PCNs placed by IRs because of infection and/or severe obstruction of the upper urinary tract, without urologic consultation or active involvement of a urologist and without taking into consideration a possible future PCNL. The placement of the preoperative emergency PCN was performed either Fluoroscopy-Guided, either UltrasoundGuided. Children under 18 years old were excluded from the study. Also, patients that were submitted to mini PCNL, patients that did not conclude the computed tomography (CT) follow-up and patients that were submitted before the operation to retrograde intrarenal surgery or ESWL were excluded from the study.

All the patients were evaluated preoperatively with a non-contrast CT and an isotopic renal scan in order to define renal anatomy and function and to plan the PCNL tract according to the stone location. Patients with positive preoperative urine cultures $\left(>10^{5} \mathrm{CFU}\right)$ were treated with antibiotics for at least 5 days before their elective procedure. Age, stone burden $\left(\mathrm{mm}^{2}\right)$, and density were recorded. The operative data included: procedure duration, preoperative PCN placed usability and reason for its non-usability. Complications categorized according to the Clavien-Dindo classification. Stone free status was assessed at 4-6 weeks follow-up visit with a non-contrast CT scan. As stone free is defined as the absence of any visible stone fragment in the non-contrast computed tomography. Informed consent was obtained from all the patients included in the study. Statistical analysis was performed by SPSS Statistics ver. 17.0 sof tware (SPSS Inc, Chicago, IL, USA). Numerical variables are presented as mean values and categorical variables are described by their absolute number and percent frequency. Mann-Whitney U-test was used to compare means of numerical variables. Chi-square test was used to compare categorical variables. All p-values were twotailed, with statistical significance set at 0.05 .

\section{The technique}

Patients in both groups were treated in prone position under general anesthesia. Flexible cystoscopy and ureteral catheter (UC) placement were performed in all patients at the beginning of the procedure. A retrograde pyelography was performed to the population without a PCN and an antegrade pyelography for the PCN patients. The insertion of and UC also to PCN patients is performed as a standard protocol to our clinic. The rationale is to be able to perform pyelography during the dilatation of the tract when the PCN is not in place anymore in case of a need. Additionally, the UC may act as a barrier to the migration of the fragments to the ureter. Also, the UC may contribute to the retrograde placement of a stent in case of a difficulty during the antegrade procedure or even to the advance of a flexible ureteroscope by the insertion of a guidewire. The PCNL operation was performed in both groups from the same experienced Endourologist (M.D.)

Standard access (Group 1) was obtained by a puncture of the desired calyx with an 18-gauge access trocar needle under multidirectional C-arm fluoroscopy guidance followed by antegrade Terumo safety guidewire insertion. A calyx is deemed as ideal for puncture according to the preoperative plan (stone burden and location) and the anatomic specifications aiming to manage the majority of the stone burden from one tract. Additionally, the puncture is directed toward the fornix of the calyx avoiding papillary damage or dilatation of the infundibulum. The usability of the PCN was assessed during the operation with the performance 
of an antegrade pyelography and the exact verification of the PCN location. If a PCN was properly located a safety wire was inserted antegradely through the tube and the nephrostomy tube was removed. Balloon tract dilatation was performed and a 30F working access sheath was placed. Ultrasonic and pneumatic lithotripsy and/or stone baskets or forceps were used also. Cases were deemed as complete when all visible stones were removed and only after the performance of a flexible nephroscopy at the end of each procedure. Nephrostomy tubes/flank positioned 18 Fr Council tip catheters were inserted in all patients, and also urethral catheters were placed. Both were placed for a minimum of 24 hours. Nephrostomy tubes were removed after an antegrade nephrostomography and patients were discharged.

Group 2 patients consisted mainly of patients originating from another institution that the PCN was placed for emergency situations and presented in the Endourology Department of Hadassah in order a PCNL to be performed. Few patients were presented in the Emergency Department of Hadassah for acute situations, such as acute renal failure and obstructive pyelonephritis and a PCN was inserted. A simple insertion of a PCN is considered a more easy procedure to perform that can quickly relieve the obstruction than to plan and perform a tract compatible to a future PCNL which demands expertise and a thorough planning and in emergency cases, the PCN insertion is the main concern.

\section{RESULTS}

Group 1 consisted of 454 patients (86.1\%) while Group 2 consisted of 73 patients (13.9\%). The mean age was 53 years for both comparison groups $(\mathrm{p}=0.733)$. The stone characteristics are depicted in Table 1 and were not statistically different between the two groups: The mean operative time $(\mathrm{p}=0.830)$, overall complication rate $(\mathrm{p}=0.859)$ and stone-free rates $(\mathrm{p}=0.453)$ are depicted in Table 2 . Skin to stone distance was significantly longer in Group 1, $142 \mathrm{~mm}$ versus $125 \mathrm{~mm}$ in Group 2 ( $\mathrm{p}<0.005)$ (Tables 1 and 2 ).

The nephrostomy location of Group 2 patients is depicted

Table 1. Preoperative data $(n=527)$

\begin{tabular}{lccc}
\hline \multicolumn{1}{c}{ Parameter } & Group 1 & Group 2 & p-value \\
\hline Number of patients & $454(86.1)$ & $73(13.9)$ & \\
Age $(\mathrm{y})$ & $52.35 \pm 3.51$ & $52.75 \pm 3.2$ & 0.733 \\
Stone burden $\left(\mathrm{mm}^{3}\right)$ & $8,490.00 \pm 45.56$ & $9,262.00 \pm 21.69$ & 0.107 \\
Hounsfield units & $1,058.00 \pm 13.42$ & $1,050.00 \pm 12.11$ & 0.953 \\
Nephrostomy location & $\mathrm{X}$ & & \\
Upper calyx & $\mathrm{X}$ & $8(11.0)$ & \\
Middle calyx & $\mathrm{X}$ & $8(11.0)$ & \\
Lower calyx & $\mathrm{X}$ & $35(47.9)$ & \\
Pelvis/Infundibulum & & $22(30.1)$ & \\
Nephrostomy usage for tract & & & \\
Yes & & 29 & $<0.005$ \\
No & 141.93 & 124.94 & \\
Skin-to-stone distance $(\mathrm{mm})$ &
\end{tabular}

Values are presented as number (\%), mean \pm standard deviation, or number only.

Table 2. Operative and postoperative data

\begin{tabular}{|c|c|c|c|}
\hline Parameter & Group 1 & Group 2 & p-value \\
\hline Procedure duration (min) & 143 & 141 & 0.830 \\
\hline Complications & $77(17.0)$ & $11(15.1)$ & 0.676 \\
\hline \multicolumn{4}{|l|}{ Complications (Clavien-Dindo classification) } \\
\hline $0-1$ & $375(82.6)$ & $62(84.9)$ & 0.859 \\
\hline$\|$ & $37(8.1)$ & $7(9.6)$ & \\
\hline IIla & $33(7.3)$ & $4(5.5)$ & \\
\hline Illb & $6(1.3)$ & $0(0.0)$ & \\
\hline IVa & $1(0.2)$ & $0(0.0)$ & \\
\hline Stone free rate (non-contrast computed tomography at $6 \mathrm{wk}$ ) & 92.9 & 92.8 & 0.453 \\
\hline
\end{tabular}

Values are presented as number only or number (\%). 
Table 3. Complication types

\begin{tabular}{lccc}
\hline \multicolumn{1}{c}{ Complication } & Group 1 & Group 2 & p-value \\
\hline Atrial fibrillation & $1(0.2)$ & $0(0.0)$ & 0.688 \\
Allergic reaction & $1(0.2)$ & $0(0.0)$ & 0.688 \\
Blood transfusion & $13(2.9)$ & $1(1.4)$ & 0.865 \\
Arterial embolization & $5(1.1)$ & $0(0.0)$ & 0.568 \\
Fever $>38^{\circ} \mathrm{C}$ & $26(5.7)$ & $2(2.7)$ & 0.807 \\
Pneumonia & $1(0.2)$ & $0(0.0)$ & 0.799 \\
Sepsis-intensive care unit & $1(0.2)$ & $0(0.0)$ & 0.799 \\
Cutaneous fistula managed with stent insertion & $2(0.4)$ & $0(0.0)$ & 0.719 \\
Hydrothorax & $4(0.9)$ & $0(0.0)$ & 0.610 \\
Obstruction managed with stent insertion & $31(6.8)$ & $0(0.0)$ & 0.145 \\
Total & & $88(16.7)$ & \\
\hline
\end{tabular}

Values are presented as number (\%).

in Table 1 . Only $29.0 \%$ of the preoperative PCNs were used for tract establishment. The reasons for not using PCN tract were: pelvic or infundibular insertion (30.0\%) and not an optimal anatomic location (70.0\%). Optimal anatomic location was considered the shortest and more straight tract dealing with the maximal stone burden.

Overall there were 88 (16.7\%) complications in the study cohort, 77 (17.0\%) and 11 (15.1\%) in Group 1 and 2, respectively ( $p=0.676$ ). The grade according to the Clavien-Dindo classification and the distribution of our complications is depicted in Table 2.

Table 3 depicts complications in details. Blood transfusion was necessary in 13 patients (2.9\%) and 1 patient (1.4\%), respectively ( $\mathrm{p}=0.865$ ). In Group 1, 5 patients were submitted to arterial embolization because of bleeding (1.1\%). Also in Group 1, 1 sepsis occurred and due to this intensive care unit care was necessary. Also, there was no statistical difference between the two groups (Table 3).

\section{DISCUSSION}

Renal access during PCNL is, most probably, the main step of the procedure with the steepest learning curve [7]. In our institution, renal access is routinely performed by the urologist in the operation theatre according to the preoperative planning. PCN however, in some special, mainly acute settings (infection, obstruction, sepsis) when there is an urgent need to drain the collecting system, is inserted by the interventional radiologist, usually without urologic guidance in view of a future PCNL. The Endourologist in several cases must decide the possible utility or not of an emergency placed PCN in a PCNL. The existence of an emergency placed PCN arises several questions and mainly whether the emergency nature of the procedure and the placement of the PCN by an interventional radiologist without taking into consideration a possible future PCNL affects the efficacy and safety of the PCNL. Recent literature data support that the preplacement of a nephrostomy tube prior a PCN neither decreased the incidence of complications and the sepsis cases nor increased the stone free rates [6]. In our study, even though all nephrostomies were inserted in an emergency setting, the patients in the 2 groups were not different in demographics or stone parameters (Tables 1 and 2). Almost half of the nephrostomies were located in the upper and middle calyceal systems or in renal pelvis or infundibulum (Table 2). The choice of calyx for the puncture is made according to the preoperative plan (stone burden and location) and the anatomic specifications. Additionally, the puncture is directed toward the fornix of the calyx avoiding papillary damage or dilatation of the infundibulum. Even though there are studies pointing out that an infundibular approach may be as safe as a fornix approach, the standard recommendation is still to puncture as anatomically as possible choosing not only general the fornix but also trying to achieve the correct location of the fornix [8-10]. A puncture through the fornix is considered the most anatomical approach since no arterial injury occurs, while in less than $8 \%$ of cases a venous injury may occur [911]. The goal of this choice is to reduce the possibility of a major vessel puncture and to obtain an optimal tract as this is presented to various anatomic and endourologic studies [9-11]. Only 29\% of preoperatively placed PCNs were used during the PCNL for the creation of the tract due to pelvic or infundibular insertion (30\%) or not optimal anatomic location (70\%) (Table 1). This emphasizes that any tract is not the right PCNL tract and of course that in the emergency cases the PCN placement was performed regardless of a possible future PCNL. A total of $70 \%$ of the nephrostomies 
were not useful for establishing a tract for the PCNL but in the emergency setting with an obstructed kidney and a septic patient the placement of a nephrostomy or a double $\mathrm{J}$ stent is considered equally effective and it is independent of a possible future PCNL procedure. A total of $30 \%$ of the PCNs were located to the pelvis or to an infundibulum but the insertion of the PCNs was not performed from a single $\mathrm{IR}$ thus the experience may be variable and additionally in acute emergency situations the first goal is to relieve the obstructed kidney without perhaps taking into consideration what would be the ideal location of the PCN in relation to a future PCNL. This, of course, indicates that is important for the urologist, either to perform by himself, either to participate in the PCN placement in the cases with a high possibility for a future PCNL management. The goal in these cases must be both the emergency decompression of the kidney, which of course is the priority and at the same time to place the $\mathrm{PCN}$ in the most appropriate tract for the future PCNL management. Moreover, there are various studies showing the efficient and safe placement from Endourologists of the PCN with results comparable to $\mathbb{R}[12,13]$ In a survey done in the United States by Bird et al. [14] it was found that only $11 \%$ of the urologists obtain the renal access during PCNL themselves. In contrary nephrostomy, presence had no effect on operation time and stone free status at the end of the procedure (Table 2). Of course, it is important to mention that both IR and Endourologists are able to place a nephrostomy tube but the different result may be a consequence of the different goal during the insertion of a PCN [14] IR place a nephrostomy in emergency cases with the goal to relieve the obstructed kidney while the puncture in the PCNL aims to succeed the ideal tract for achieving a stone-free status. At the same time, Urologists should also be capable of placing a nephrostomy tube in emergency cases. The ideal of course scenario would be regardless if an IR or an Endourologist is placing the PCN in the emergency cases the procedure to be performed taking into consideration the possibility of a future PCNL in conjunction with the characteristics of the stone. The presence of a PCN that is not useful for the establishment of a tract, necessitates a new puncture, with additional risks and prolonging the operation. Even though $71 \%$ of the patients of Group 2 required a new puncture in order to perform the PCNL successfully the complications were not statistically different in both groups.

Of course, the limited number of Group 2 patients could influence the statistical significance. The complications between the two groups were not statistically significant despite that the PCNs were placed in emergency cases and even though that Group 1 patients were more obese, and additionally had a higher skin to stone distance constituting tract establishment perhaps more demanding (Tables 1-3). Patel et al. [15] showed that the obtained access from the urologist is effective especially through the upper pole, which could be a subject of hesitancy for the interventional radiologist. Additionally it has an acceptable complication risk profile even in the management of a large stone burden [15]. In our study the majority of the IIIb and IVa ClavienDindo grade complications occurred in Group 1, suggesting a possible protective role of the nephrostomy. It is important to mention that although not statistically significant the majority of the cases that required blood transfusion was in Group 1. Also only in this group occurred the need of arterial embolization indicating again the possible protection from a preoperative established tract. There was also no statistical significance in septic complications between the groups, even though the absence of any preoperative drainage in Group 1 could theoretically constitute more susceptible to urosepsis this population. An interesting result from our study is the occurrence of a cutaneous fistula. Although the placement of a PCN created an initial tract lasting significantly longer period there was not an increase in the occurrence of a cutaneous fistula in this group. Also, it is important to mention that the stone-free rates between the 2 groups did not show a statistically significant difference.

Our limitations are that this is a retrospective study without randomization since all the patients with a PCN were emergency cases. Also, the placement of the PCNs is quite random since it was performed by an interventional radiologist without urologic tract planning. Thus, the results may not reflect the true impact of PCN. A properly placed nephrostomy could positively affect the procedure in terms of accuracy, bleeding, operation time and stone approach. To our knowledge this the first study dealing with the utility and safety of an emergency placed PCN as a possible tract for an upcoming PCNL. Of course, new studies are needed in order to evaluate the preoperatively placed PCN and its effects to PCNL results.

\section{CONCLUSIONS}

A PCN placed in emergency cases from an IR without urologic consultation may lead to additional renal punctures during an upcoming PCNL in order to establish a correct tract for the management of the stone burden. PCN presence before PCNL does not increase complication rates, the time of the procedure and does not affect the stone-free rates. It is important for the urologists to be involved in the 
emergency placed PCNs in cases with a high possibility for

a PCNL procedure in the near future.

\section{CONFLICTS OF INTEREST}

The authors have nothing to disclose.

\section{REFERENCES}

1. Patel SR, Nakada SY. The modern history and evolution of percutaneous nephrolithotomy. J Endourol 2015;29:153-7.

2. Tepeler A, Armağan A, Akman T, Polat EC, Ersöz C, Topaktaş $\mathrm{R}$, et al. Impact of percutaneous renal access technique on outcomes of percutaneous nephrolithotomy. J Endourol 2012;26: 828-33.

3. Lashley DB, Fuchs EF. Urologist-acquired renal access for percutaneous renal surgery. Urology 1998;51:927-31.

4. Dagli M, Ramchandani P. Percutaneous nephrostomy: technical aspects and indications. Semin Intervent Radiol 2011;28:424-37.

5. Tomaszewski JJ, Ortiz TD, Gayed BA, Smaldone MC, Jackman SV, Averch TD. Renal access by urologist or radiologist during percutaneous nephrolithotomy. J Endourol 2010;24:1733-7.

6. Bearelly P, Lis C, Trussler J, Katz MH, Babayan RK, Wang DS. Nephrostomy tube placement prior to percutaneous nephrolithotomy does not impact outcomes. Can J Urol 2018;25:9497-
502

7. de la Rosette JJ, Laguna MP, Rassweiler JJ, Conort P. Training in percutaneous nephrolithotomy-a critical review. Eur Urol 2008;54:994-1001.

8. Kallidonis P, Kyriazis I, Kotsiris D, Koutava A, Kamal W, Liatsikos E. Papillary vs nonpapillary puncture in percutaneous nephrolithotomy: a prospective randomized trial. J Endourol 2017;31(S1):S4-9.

9. Sampaio FJ. Renal anatomy. Endourologic considerations. Urol Clin North Am 2000;27:585-607.

10. Miller NL, Matlaga BR, Lingeman JE. Techniques for fluoroscopic percutaneous renal access. J Urol 2007;178:15-23.

11. Sampaio FJ, Zanier JF, Aragão AH, Favorito LA. Intrarenal access: 3-dimensional anatomical study. J Urol 1992;148:1769-73.

12. Masood J, Yeo L, Zaman F, El-Husseiny T, Moraitis K, Maan $Z$, et al. Should urologists in the UK undertake their own nephrostomies and renal access for endourological procedures: what does the future hold? BJU Int 2009;104:755-7.

13. Rana AM, Zaidi Z, El-Khalid S. Single-center review of fluoroscopy-guided percutaneous nephrostomy performed by urologic surgeons. J Endourol 2007;21:688-91.

14. Bird VG, Fallon B, Winfield HN. Practice patterns in the treatment of large renal stones. J Endourol 2003;17:355-63.

15. Patel AP, Bui D, Pattaras J, Ogan K. Upper pole urologistobtained percutaneous renal access for PCNL is safe and efficacious. Can J Urol 2017;24:8754-8. 